\title{
Comparison of Frequency Features of Pulse Echoes from Different Reflectors
}

\author{
Anatoly B. Rinkevich, Olga V. Nemytova, and Dmitry V. Perov \\ Ural Division of RAS, Institute of Metal Physics, 18 S. Kovalevskaya Street, Ekaterinburg 620041, Russia \\ Correspondence should be addressed to Dmitry V. Perov, peroff@imp.uran.ru
}

Received 4 August 2011; Accepted 29 August 2011

Academic Editor: J. Hu

Copyright () 2011 Anatoly B. Rinkevich et al. This is an open access article distributed under the Creative Commons Attribution License, which permits unrestricted use, distribution, and reproduction in any medium, provided the original work is properly cited.

\begin{abstract}
The analysis of interaction of ultrasonic pulses with different reflectors which are located in elastic media is carried out. A parameter which allows estimating the form of reflector is instantaneous frequency of pulse-echo. This parameter is an unsteady pulse echo characteristic. Instantaneous frequency determination algorithm is to use wavelet transformation which has increased noise immunity. It is shown that the instantaneous frequency value for a pulse echo is often shifted from a rated frequency of piezoelectric transducer. Difference between the frequencies extensively depends on the reflector form.
\end{abstract}

\section{Introduction}

At the present time, the problem of determination of the form and the size of latent defect in goods is very urgent problem. Solution of this problem is of great practical interest at the quite a number industry, medicine, and geophysics. In general, the delay time and the amplitude of pulse echo are the main informative parameters in ultrasonic nondestructive testing. Unfortunately, these parameters do not allow, in all instances, drawing definite conclusion about size and form of defect.

In principle, a defect form reconstruction is carried out by using a computer tomography techniques which are more expensive and time-consuming techniques and which require the easy access to the detected object. In some cases, however, it is necessary to know only a class of reflecting object which a defect belongs to. The problem can be solved by using the only transducer placed at a chosen position. In this case, it can be considered a question about the finding of determined informative features which allow classifying the defect.

Nowadays a number of ultrasonic techniques of defecttype determination exist [1-4]. The methods, to some extent, allow judging about the defect form. But they have some limitation. Along with the existing techniques we suggest our method which is to analyse the time dependence of the instantaneous frequency of the reflected signal. For the continuous sinusoidal signal the instantaneous frequency is a constant value. Whereas, for pulsed signal, the instantaneous frequency can be various at the different moments of time inside the impulse. The differences are originated from diffraction influence and the frequency dependency of attenuation coefficient.

The frequency of ultrasonic vibrations can be measured with radioengineering methods via analysis of the spectrum of an echo signal received by a transducer or via measurements of the vibration period in echo pulses performed with a broadband oscilloscope. The effective frequency $f_{\text {eff }}$ of a transducer's echo pulse is found from the formula

$$
f_{\text {eff }}=\frac{n}{2 \tau}
$$

where $n$ is the number of half periods in the echo pulse (of positive and negative polarities) and $\tau$ is the total duration of these half periods. Note that some papers (e.g., $[5,6]$ ) point to the fact that the aforementioned procedures for determining the frequency are not always adequate for practical situations and the results obtained through them may be different from each other. In our opinion, the techniques currently employed to determine the vibration frequencies during ultrasonic NDT do not adequately take into account the peculiarities of real echo pulses reflected by 
flaws of different types. Because such signals often have nonstationary characteristics, namely, their dominant frequency changes with time inside the pulse and the envelope often has an intricate profile, their description requires the use of such analytic methods that allow the frequency parameters of signals to be estimated at different times. Determining the instantaneous frequency of a signal on the basis of an analytic signal concept can be regarded as one of such methods $[7,8]$. Because the practical application of this approach is limited by its very low noise immunity, we have proposed a modified version of this technique based on regularization of the procedure for determining the instantaneous signal frequency with the use of the wavelet transform. As is shown below, this regularization ensures an increased noise immunity of this procedure.

Let us now describe in detail, in the second part of the paper, the determination algorithms of instantaneous frequency based the applying wavelet transformation. An experimental technique is considered in the third part of the paper. The descriptions of experimental samples, piezoelectric transducers (PETs), and digital flaw detector which radiate direct impulse are cited. The results obtained and discussion of the results is considered in the fourth part of the paper. In the last part of the paper the general conclusions are made.

\section{Algorithms for Determining the Instantaneous Frequency}

Let there be some analyzed signal $s(t)$ that depends on time $t$. Complex analytic signal $\dot{z}(t)$ corresponding to the said analyzed signal can be obtained with the use of Hilbert transform $H[s(t)][7,8]$ :

$$
\dot{z}(t)=s(t)+i H[s(t)]
$$

where

$$
H[s(t)]=\frac{1}{\pi} \int_{-\infty}^{+\infty} \frac{s(\tau)}{t-\tau} d \tau .
$$

Instantaneous frequency $\tilde{f}(t)$ of signal $s(t)$ can be found from the formula $[7,8]$ :

$$
\tilde{f}(t)=\operatorname{Im}\left[\frac{1}{\dot{z}(t)} \frac{d \dot{z}(t)}{d t}\right] .
$$

As an example of using this relationship, let us consider a signal with a Gaussian envelope whose angular frequency increases with time according to the linear law $\omega(t)=\omega_{0}+\mu t$

$$
s(t)=S_{0} \exp \left(-\beta t^{2}\right) \cos \left(\omega_{0} t+\mu t^{2}\right),
$$

where $S_{0}$ is the signal amplitude, $\beta$ is the factor determining the signal duration, $\omega_{0}$ is the value of the angular frequency at moment $t=0$, and $\mu$ is the frequency deviation. The signal defined by formula (5) is shown in Figure 1(a) for $S_{0}=1, \beta=3 \cdot 10^{3}, f_{0}=\omega_{0} / 2 \pi=5 \mathrm{MHz}, \mu=5$. $10^{8} \mathrm{rad} / \mathrm{s}^{2}$. Figure $1(\mathrm{~b})$ shows that the result of calculating the instantaneous signal frequency from formula (4) fully coincides with the dependence

$$
f(t)=\frac{\omega_{0}+\mu t}{2 \pi}
$$

proposed above. Note that, because formula (4) contains a differentiation operator, the calculation result strongly depends on the noise present in the analyzed signal. More stable results can be attained with the use of modified formula (4) resulting from the use of a continuous wavelet transforms [9].

The wavelet spectrum of analytic signal $\dot{z}(t)$ can be found with the help of either of the two equivalent relationships [10, 11]

$$
\dot{W}_{z}(\xi, \theta)=\frac{1}{\sqrt{|\xi|}} \int_{-\infty}^{+\infty} \dot{z}(t) \stackrel{*}{\psi}\left(\frac{t-\theta}{\xi}\right) d t
$$

or

$$
\dot{W}_{z}(\xi, \theta)=\frac{\sqrt{|\xi|}}{2 \pi} \int_{-\infty}^{+\infty} \dot{Z}(\omega) \stackrel{*}{\Psi}(\xi \omega) \exp (i \theta \omega) d \omega
$$

where $\dot{\psi}(t)$ is a wavelet, $\dot{\Psi}(\omega)$ and $\dot{Z}(\omega)$ are the Fourier spectra corresponding to wavelet $\psi(t)$ and analytic signal $\dot{z}(t), \xi$ is the scale factor, and $\theta$ is the shift parameter.

Using the known spectrum $\dot{W}_{z}(\xi, \theta)$ of the analytic signal, one can obtain the expression for the instantaneous frequency:

$$
\tilde{\widetilde{f}}\left(\theta, \xi^{*}\right)=\frac{1}{2 \pi} \operatorname{Im}\left[\frac{1}{\dot{W}_{z}\left(\xi^{*}, \theta\right)} \frac{d \dot{W}_{z}\left(\xi^{*}, \theta\right)}{d \theta}\right],
$$

where $\xi^{*}=\xi^{*}(\theta)$ is the value of scale factor $\xi$ corresponding to the maximum module of the wavelet spectrum of the analytic signal, that is, to the condition $\left|\dot{W}_{z}\left(\xi^{*}, \theta\right)\right|=$ $\max \left\{\left|\dot{W}_{z}(\xi, \theta)\right|\right\}$, where $\theta$ is the shift parameter of the wavelet transform, a variable that is analogous in the sense of variable $t$ in formula (4). Figure 1(b) presents the results obtained with the use of formula (9) for signal (5) with the parameters $\alpha=3 \cdot 10^{3} \mathrm{~s}^{-1}, f_{0}=\omega_{0} / 2 \pi=5 \mathrm{MHz}$, and $\mu=5 \cdot 10^{8} \mathrm{rad} / \mathrm{s}^{2}$.

The MHAT wavelet $[10,11]$

$$
\psi(t)=\frac{2}{\sqrt{3 \sqrt{\pi}}}\left(1-t^{2}\right) \exp \left(-\frac{1}{2} t^{2}\right)
$$

was used to calculate the wavelet spectrum from formula (7).

Figure 1(c) shows, respectively, the modulus of the wavelet spectrum of the analytic signal in the form of level lines. Note that the time plotted along the abscissa axes in these two figures corresponds to shift parameter $\theta$ and that the frequencies plotted along the ordinate axes are recalculated on the basis of scale factor $\xi$ from the formula $[10,11] f=(1 / \pi \xi) \sqrt{5 / 8}$. Values of scale factor $\xi$ correspond to the condition $\left|\dot{W}_{z}\left(\xi^{*}, \theta\right)\right|=\max \left\{\left|\dot{W}_{z}(\xi, \theta)\right|\right\}$. According to Figure 1(b), the values of the instantaneous frequency calculated from formulas (4) and (9) coincide quite well: the difference between them is less than $0.5 \%$. 


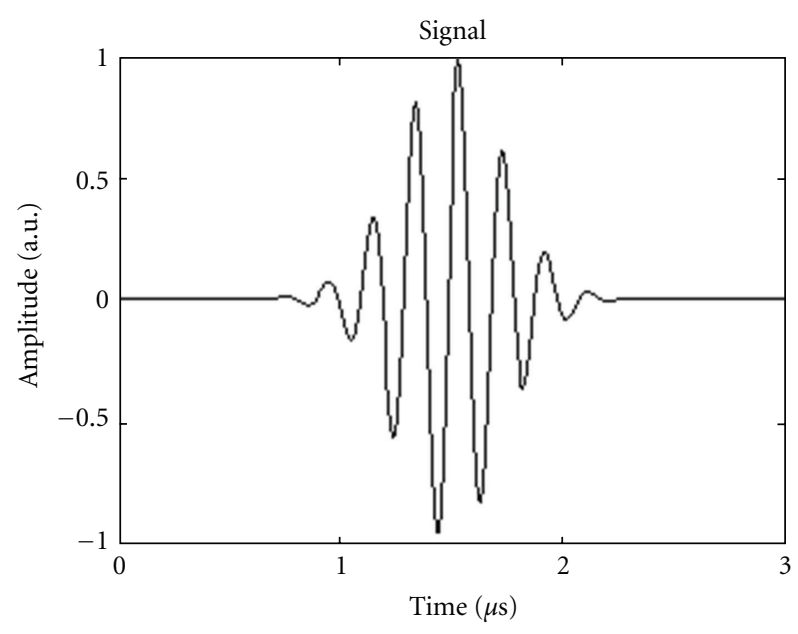

(a)

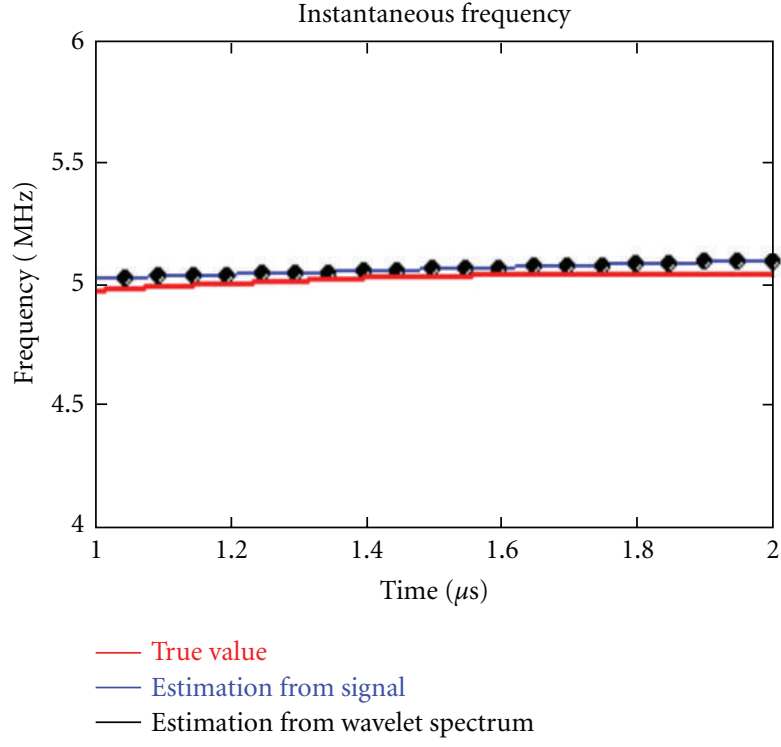

(b)

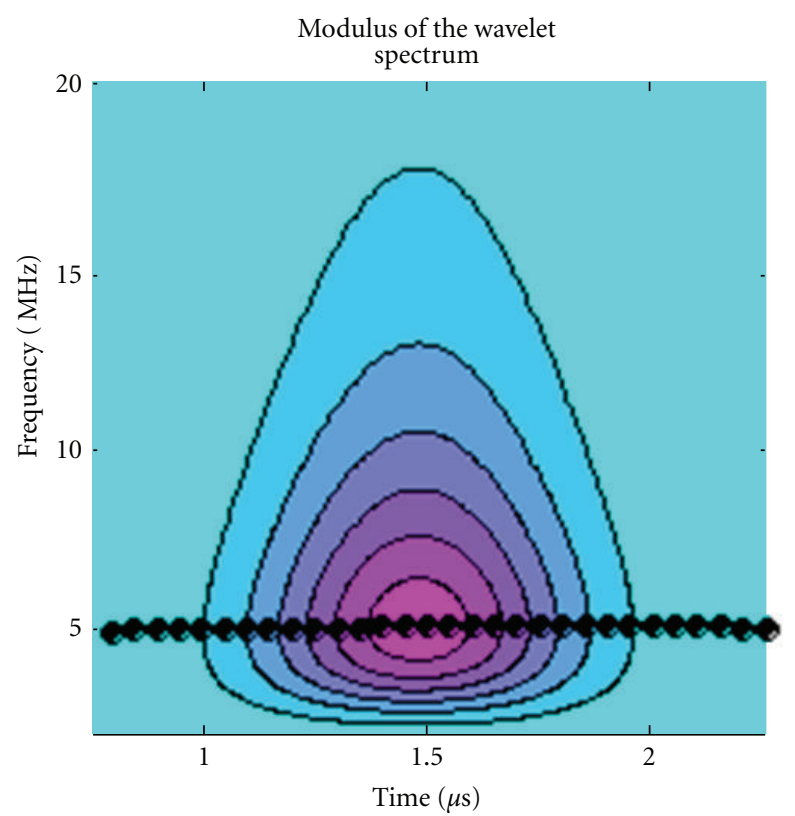

(c)

FIGURE 1: Evaluation of the instantaneous frequency of a linearly frequency-modulated (LFM) signal with a Gaussian envelope in the absence of noise: (a) analyzed signal; (b) time dependences of the instantaneous frequency obtained directly from the analytic signal (thin blue line) and from the wavelet spectrum of the analytic signal (bold red line) in comparison to the theoretical dependence (dots); (c) modulus of the wavelet spectrum of the analytic signal, whose maxima corresponding to different moments are marked with dots.

Let us now compare two algorithms from the standpoint of their stability with respect to the effect of noise. To do this, we add additive white Gaussian noise to signal (2) and execute algorithms (10) and (5). The results obtained for the signal-to-noise ratios of 40 and $6 \mathrm{~dB}$ are shown in Figures 2 and 3, respectively. Comparison of these figures to Figure 1 clearly demonstrates both the high noise immunity of the algorithm for estimating the instantaneous frequency with the use of the wavelet spectrum and the absence of such immunity in the classical algorithm defined by formula (4).

\section{Experimental Technique}

Experimental investigations were carried out by using the steel samples which have various set of the artificial reflectors. Several types of reflectors were chosen, in particular, notches with various area of flat face from $0.8 \mathrm{~mm}^{2}$ to $8.75 \mathrm{~mm}^{2}$, interfacial angle with various opening angles from $56^{\circ}$ to $90^{\circ}$, flat-bottomed drilling either with various area of flat reflecting surface from $2 \mathrm{~mm}^{2}$ to $6 \mathrm{~mm}^{2}$ which are located in the same depth of $32 \mathrm{~mm}$ or with the same area of flat 


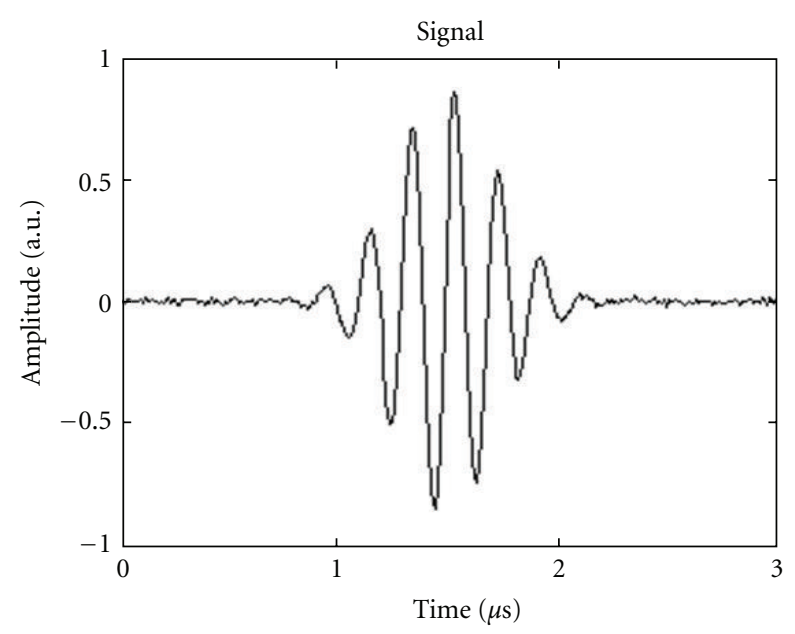

(a)

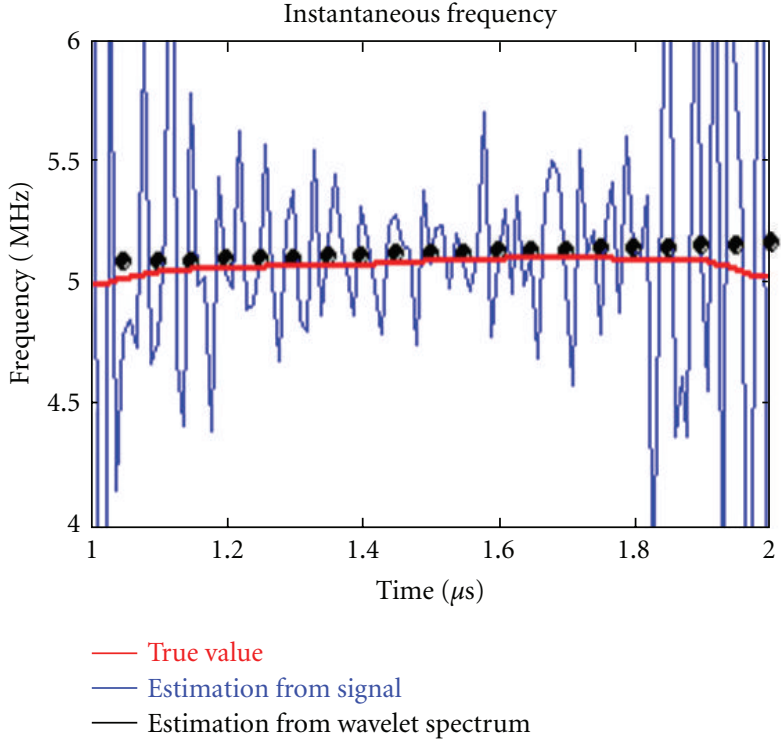

(b)

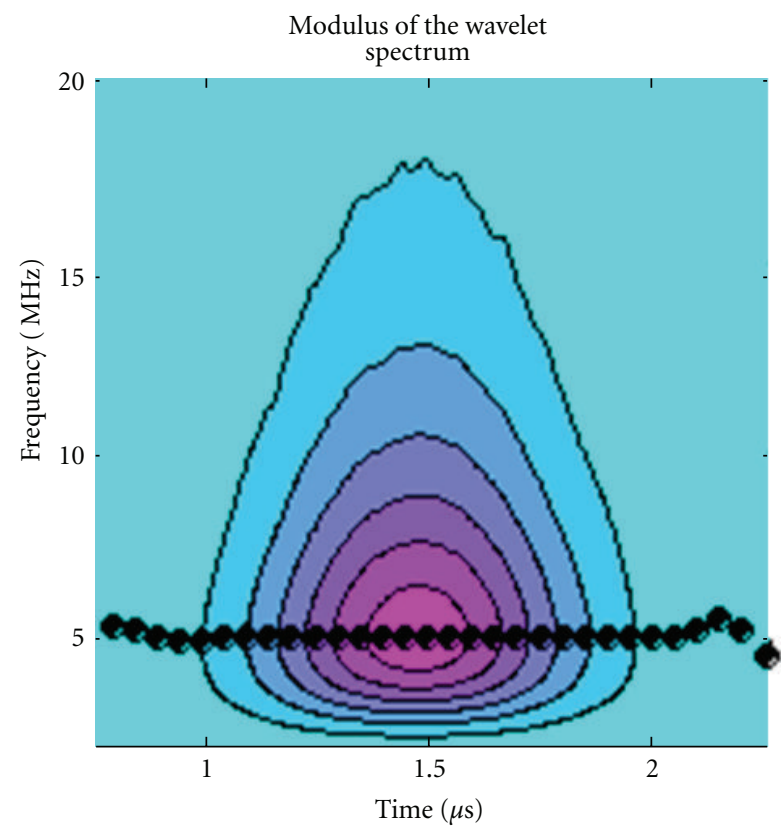

(c)

Figure 2: Evaluation of the instantaneous frequency of an LFM signal with a Gaussian envelope in the presence of additive white Gaussian noise (at a signal-to-noise ratio of $40 \mathrm{~dB}$ ): (a) analyzed signal and (b) time dependences of the instantaneous frequency that are obtained directly from the analytic signal (thin blue line) and from the wavelet spectrum of the analytic signal (bold red line) in comparison to the theoretical dependence (dots); (c) modulus of the wavelet spectrum of the analytic signal, whose maxima corresponding to different moments are marked with dots.

reflected surface of $2 \mathrm{~mm}^{2}$ which are located in various depths from $27 \mathrm{~mm}$ to $52 \mathrm{~mm}$, and the set of through-hole drilling either with various diameters from $2 \mathrm{~mm}$ to $6 \mathrm{~mm}$ which are located in the same depth of $30 \mathrm{~mm}$ or with the same diameter of $2 \mathrm{~mm}$ which are located in various depth from $45 \mathrm{~mm}$ to $25 \mathrm{~mm}$ (see Figure 4). In the investigation, a digital flaw detector PCUS-10 is used to radiate direct impulses and resave the pulse echoes. The angle piezoelectric transducers with the rated frequency of $5 \mathrm{MHz}$ and with the incidence angle of $40^{\circ}$ and the direct piezoelectric transducer with the rated frequency of $4 \mathrm{MHz}$ are used to radiate and resave ultrasonic waves. The transducers are combined; that is, a piezoelectric element acts at first as a radiator and then as a receiver of ultrasonic oscillations.

The measurements, for each of transducers, were carried out in the following way. The transducer was located on 


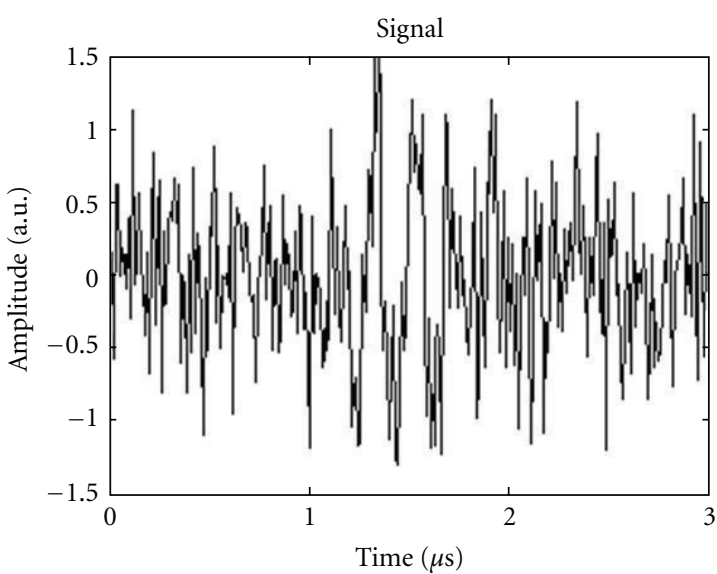

(a)

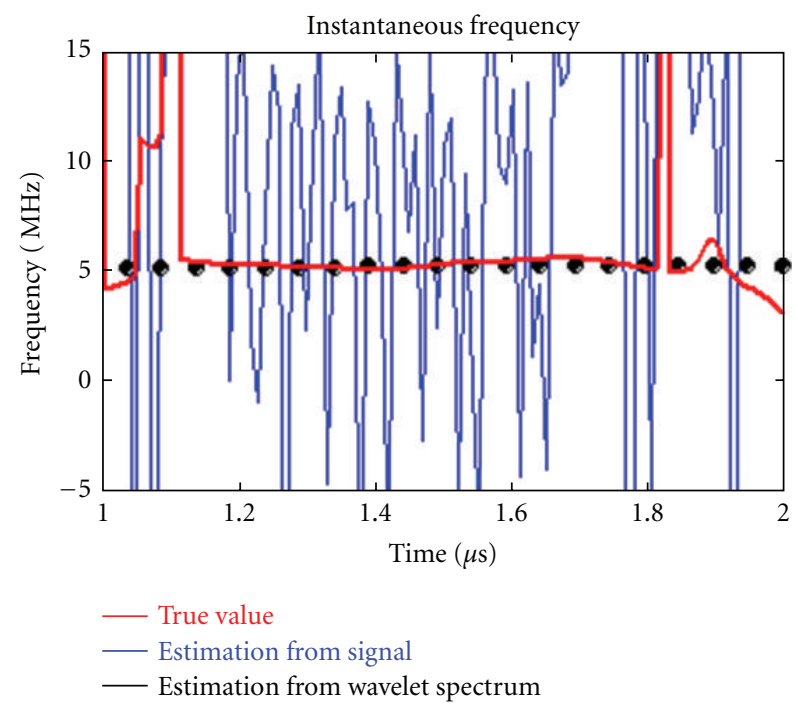

(b)

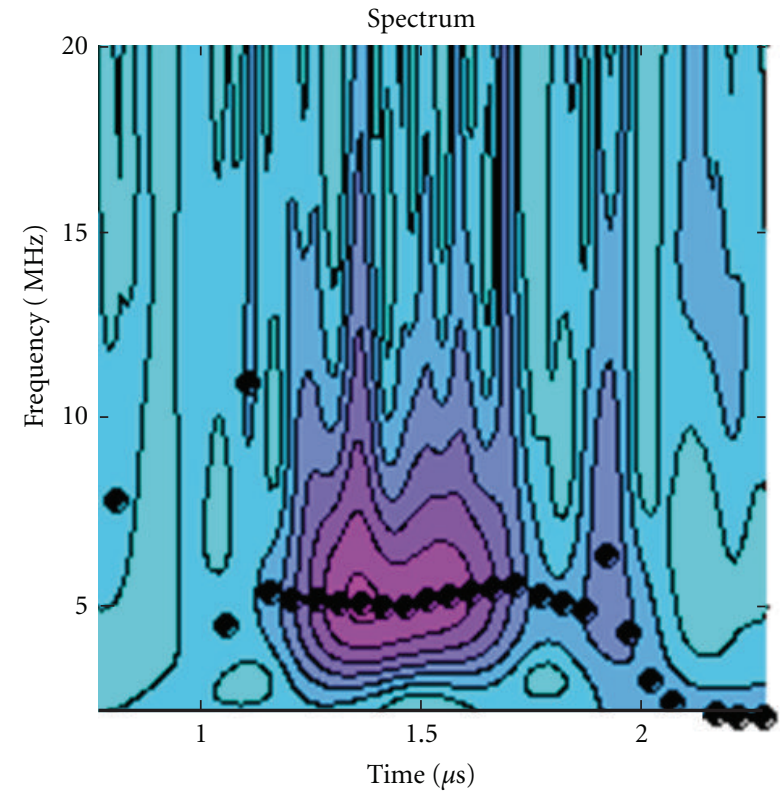

(c)

FIGURE 3: Evaluation of the instantaneous frequency of an LFM signal with a Gaussian envelope in the presence of additive white Gaussian noise (at a signal-to-noise ratio of $6 \mathrm{~dB}$ ): (a) analyzed signal and (b) time dependences of the instantaneous frequency that are obtained directly from the analytic signal (thin blue line) and from the wavelet spectrum of the analytic signal (bold red line) in comparison to the theoretical dependence (dots); (c) modulus of the wavelet spectrum of the analytic signal, whose maxima corresponding to different moments are marked with dots.

the middle line of sample surface in the point where pulse echo amplitude was maximal. In the point, A-type scan was received and saved in the memory of PC to be treated and analyzed.

The pulse echo envelope is shown schematically in Figure 5. The values of instantaneous frequency of pulse echo $f_{1}, f_{2}$, and $f_{0}$ at the moments of time corresponding $t_{1}, t_{2}$ и $t_{0}$ points are found via analyzing the signals obtained by using the wavelet algorithm of frequency determination.

\section{Results of Ultrasonic Measurements and Discussion}

One of the aims of the investigation is to obtain dependences of instantaneous frequency values $f_{\mathrm{M}}$ at the selected moments of time on few parameters: the diameter of reflector, and the depth of reflector's location, the area of reflector, the opening angle of interfacial angle. Thus, the instantaneous frequency is determined here at the $t_{1}, t_{2}$ и $t_{0}$ 


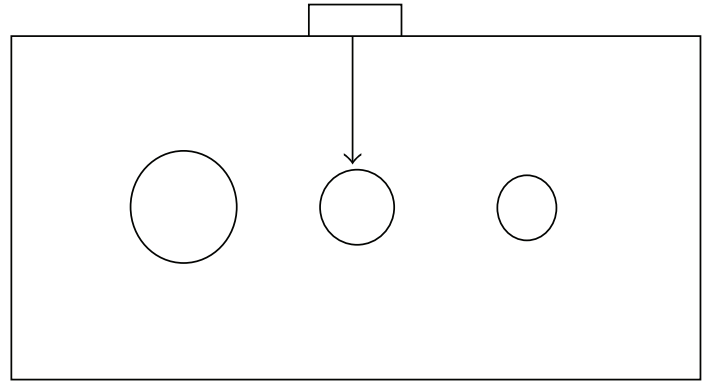

(a)

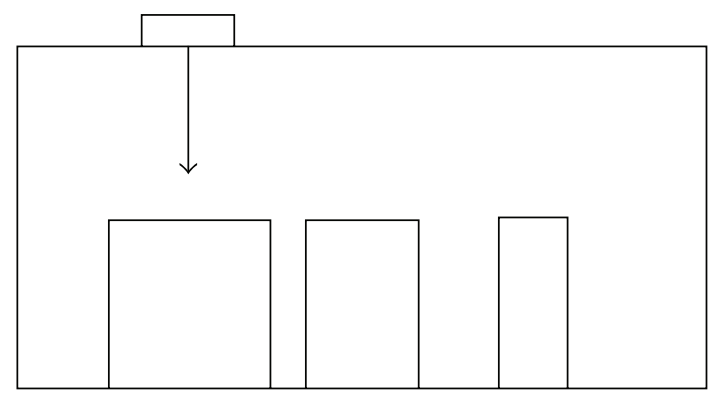

(c)

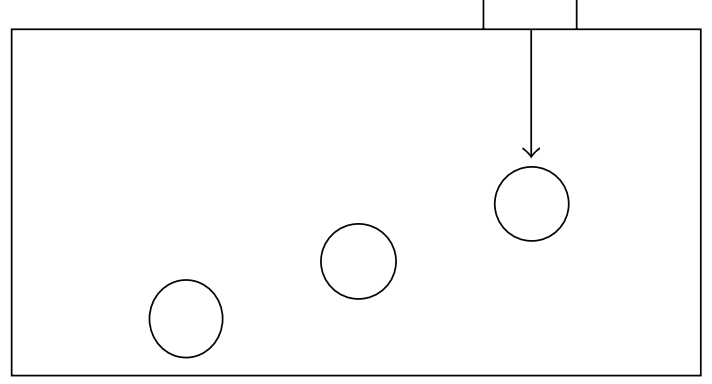

(b)

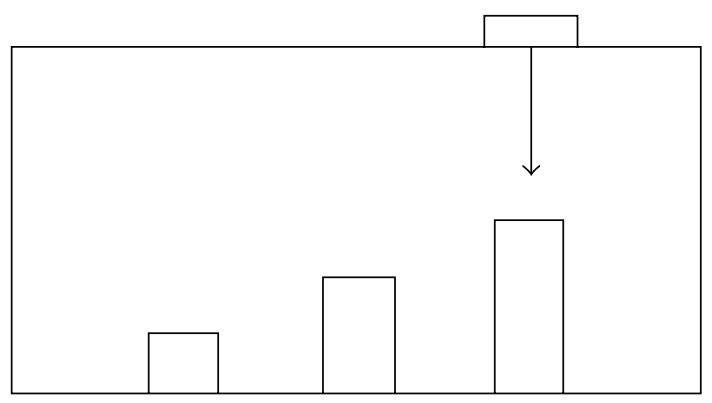

(d)

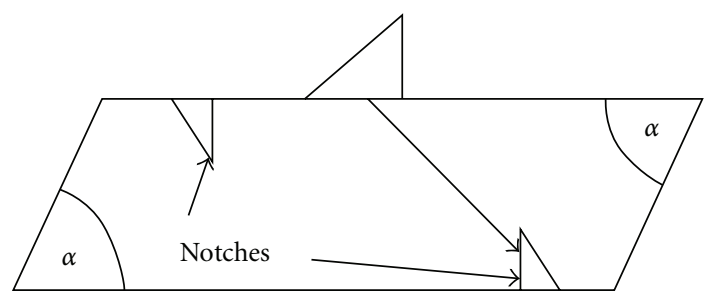

(e)

FIGURE 4: The samples investigated with artificial reflectors: (a) through-hole drillings with various diameter, which are located in the same depth; (b) through-hole drillings with the same diameter which are located in various depth; (c) flat-bottomed drillings with various diameter which are located in the same depth; (d) flat-bottomed drillings with the same diameter which are located in various depth; (e) the set of samples with notches which have various area of flat face and with interfacial angles which have various opening angles.

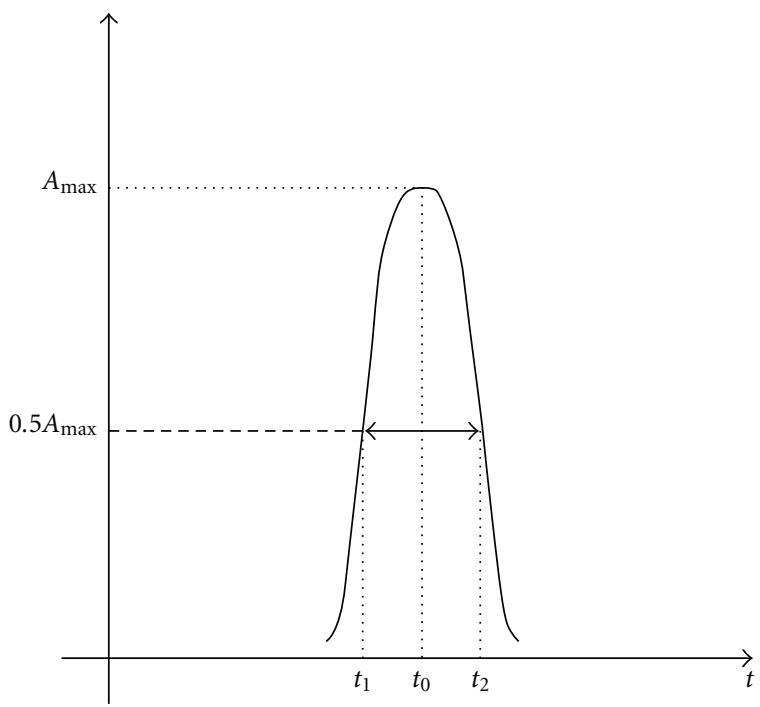

Figure 5: A pulse echo envelope. points. The dependences of $f_{\mathrm{M}}(d), f_{\mathrm{M}}(h), f_{\mathrm{M}}(S), f_{\mathrm{M}}(\alpha)$ are shown in Figure $6, d$ is the diameter of flat-bottomed drilling or through-hole drilling, $h$ is the distance between transducer and reflector, $S$ is the area of flat face notch, and $\alpha$ is the opening angle of interfacial angle.

From graphs presented in Figure 6 some deductions can be concluded. In the first place, from the graphs one can see that instantaneous frequency $f_{0}$ which is considered at a maximum of envelope of signal analysed for the notches reflectors and interfacial angles reflectors exceeds instantaneous frequencies $f_{1}$ and $f_{2}$ which are considered at the moments of time where the envelope amplitude equals one half of its maximum value. In turn, for the flat-bottomed drilling reflectors and for the through-hole drilling reflectors the value $f_{0}$ is located between the values of $f_{1}$ and $f_{2}$. In the second place, for the notches reflectors, the value $f_{0}$ approximately equals or even exceeds the rated frequency of transducer. But for flat-bottomed drillings and for throughhole drillings the value $f_{0}$ is below the rated frequency of transducer. 


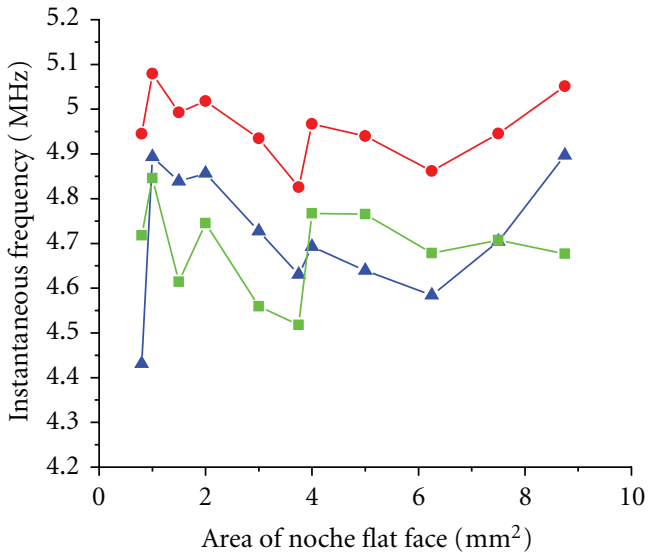

(a)

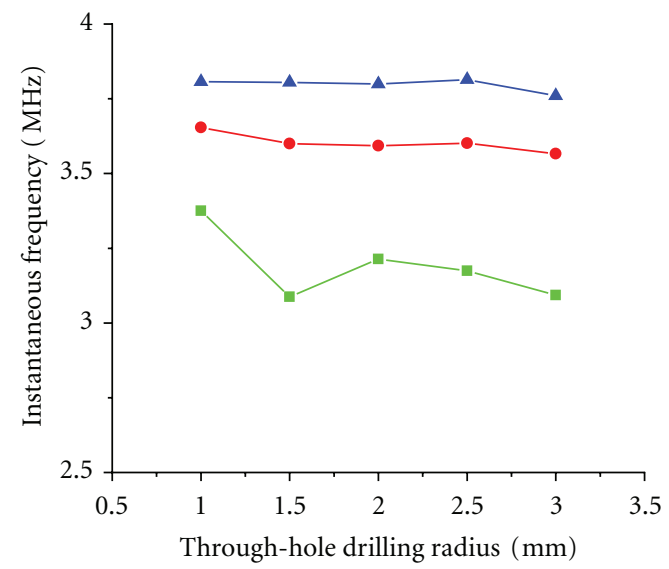

(c)

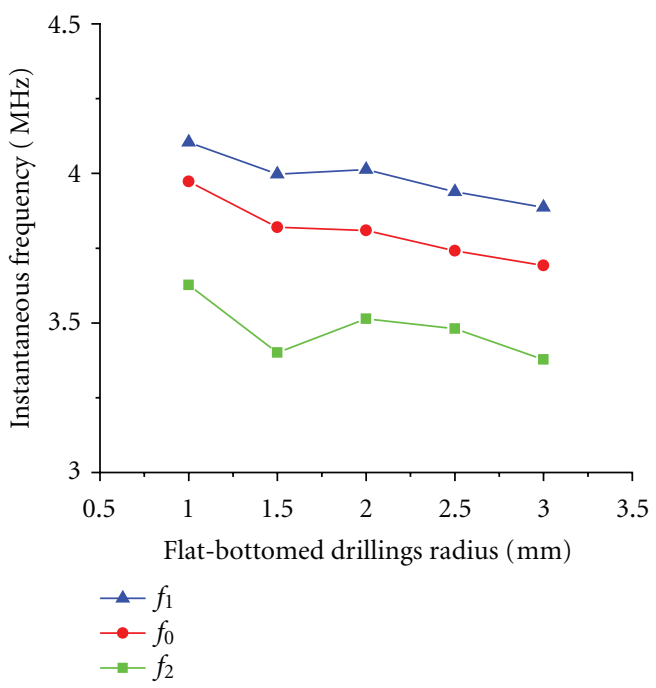

(e)

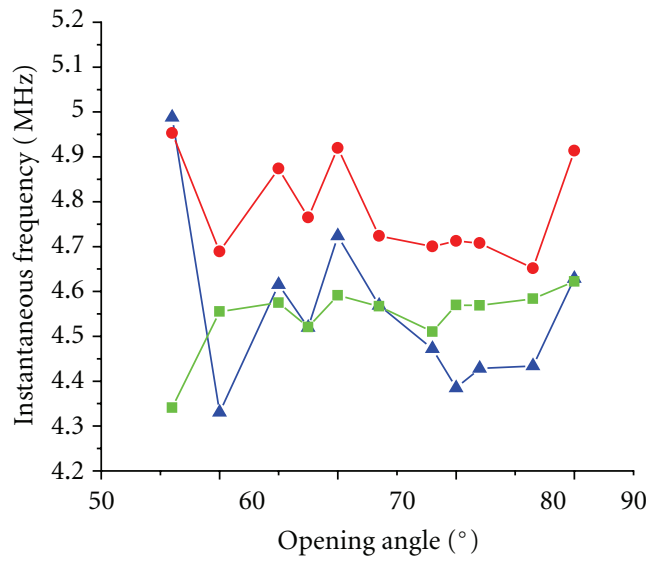

(b)

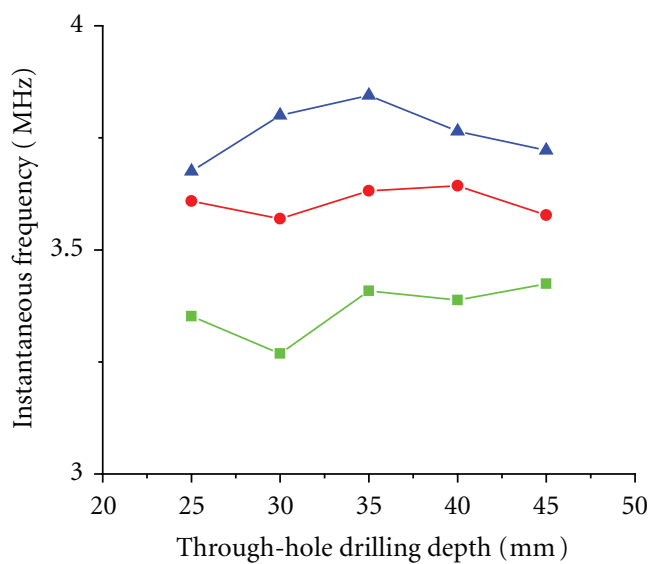

(d)

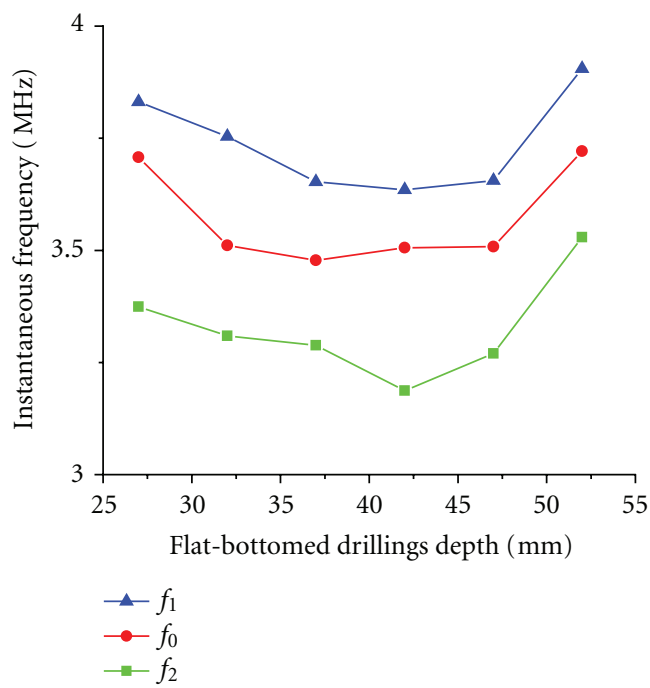

(f)

FIGURE 6: A relation of instantaneous frequency to the reflector parameters: (a) notches which have various area of flat face; (b) interfacial angles which have various opening angles; (c) through-hole drillings with various diameter, which are located in the same depth; (d) throughhole drillings with the same diameter which are located in various depth; (e) flat-bottomed drillings with various diameter which are located in the same depth; (f) flat-bottomed drillings with the same diameter which are located in various depth. 


\section{Conclusions}

The algorithm for instantaneous frequency determination based on the using of continuous wavelet transformation was worked out. Applying this algorithm, one can increase the noise immunity. The algorithm was applied to the signals obtained from the reflectors which have different shape in order to calculate the instantaneous frequency in different moments within the ultrasonic echo pulse.

The results obtained argue that instantaneous frequency of pulse echo is significantly changed depending on the type of reflector. It was ascertained that the change depends on the moment of the pulse echo envelope region to which the measurement is corresponded. In some cases the form of reflectors determines the frequency change and the correspondence between the instantaneous frequency and the rated frequency of transducer. For instance, from our results it follows that for plane reflectors the instantaneous frequency obtained for a moment where the magnitude of the envelope is maximal exceeds the frequencies at moments where the magnitude is one half of the maximal value. The results obtained are the beginning of studies of the possibility of using the instantaneous frequency estimation for classifying the echo signals reflected from different defects.

\section{Acknowledgment}

The work is carried out following to RAS theme no. 01.2.006 13393 with partial support from the RAS program "Diagnostics".

\section{References}

[1] V. G. Scherbinskyi and V. E. Belyi, "New informative feature of defects nature under the ultrasonic testing," Defectoskopiya, no. 3, pp. 27-36, 1975.

[2] I. S. Whittaker and T. J. Iessop, "Ultrsonic detection and measurements of defects in stain-less steel," Journal of Nondestructive Testing, vol. 23, no. 6, pp. 293-303, 1981.

[3] A. K. Gurvich and L. I. Kuzmina, "Scattering indicatrix as the additional information source about defect revealed," Defektoskopiya, no. 6, pp. 47-56, 1970.

[4] S. P. Perevalov, "Measurement assurance of nondestructive testing: II. Comparative analysis of various methods for the production of compact angular reflectors," Defektoskopiya, no. 9, pp. 32-40, 1994.

[5] A. A. Shcherbakov and V. N. Danilov, "Definition of effective frequency of echo-pulse of ultrasonic transducers," Defektoskopiya, no. 4, pp. 23-31, 1998.

[6] V. N. Danilov, I. N. Ermolov, and A. A. Shcherbakov, "Determination of the vibration frequency in nondestructive testing," Russian Journal of Nondestructive Testing, vol. 39, no. 3, pp. 171-178, 2003.

[7] L. A. Weinstein and D. E. Vakman, Frequencies Separation in Theory of Oscillations and Waves, Nauka, Moscow, Russia, 1983.

[8] D. V. Perov, A. B. Rinkevich, and O. V. Nemytova, "Interaction of pulse ultrasonic signals with reflectors of different types," Russian Journal of Nondestructive Testing, vol. 43, no. 6, pp. 369-377, 2007.
[9] D. V. Perov and A. B. Rinkevich, "Using the different types of wavelet transform for ana-lyzing acoustic fields and signals in elastic media," in New Research on Acoustics, B. N. Weiss, Ed., pp. 65-109, Nova Science Publishers, Hauppauge, NY, USA, 2008.

[10] D. V. Perov, A. B. Rinkevich, and O. A. Kusonskii, "A wavelet analysis of super-long-period seismic oscillations," in Sbornik trudov XVI Sessii Rossiiskogo akusticheskogo obshchestva (Proceedings of 16th Session of the Russian Acoustic Society), vol. 1, pp. 279-282, GEOS, Moscow, Russia, 2005.

[11] D. V. Perov, A. B. Rinkevich, Ya. G. Smorodinskii, and B. Keler, "Using wavelets for analyzing ultrasonic fields detected by a laser interferometer. Flaw detection and localization in an aluminum single-crystal," Russian Journal of Nondestructive Testing, vol. 37, no. 12, pp. 889-899, 2001. 

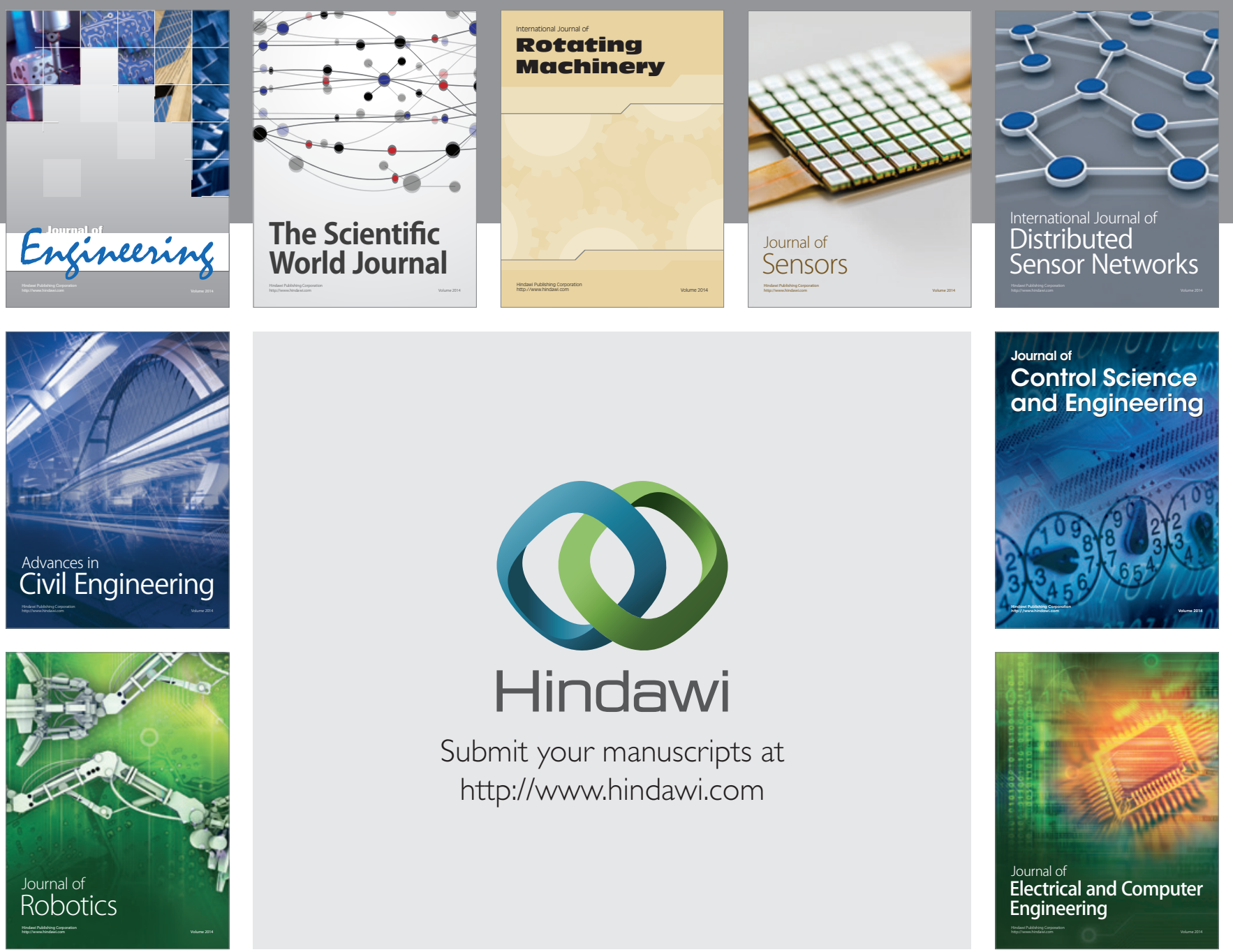

Submit your manuscripts at

http://www.hindawi.com
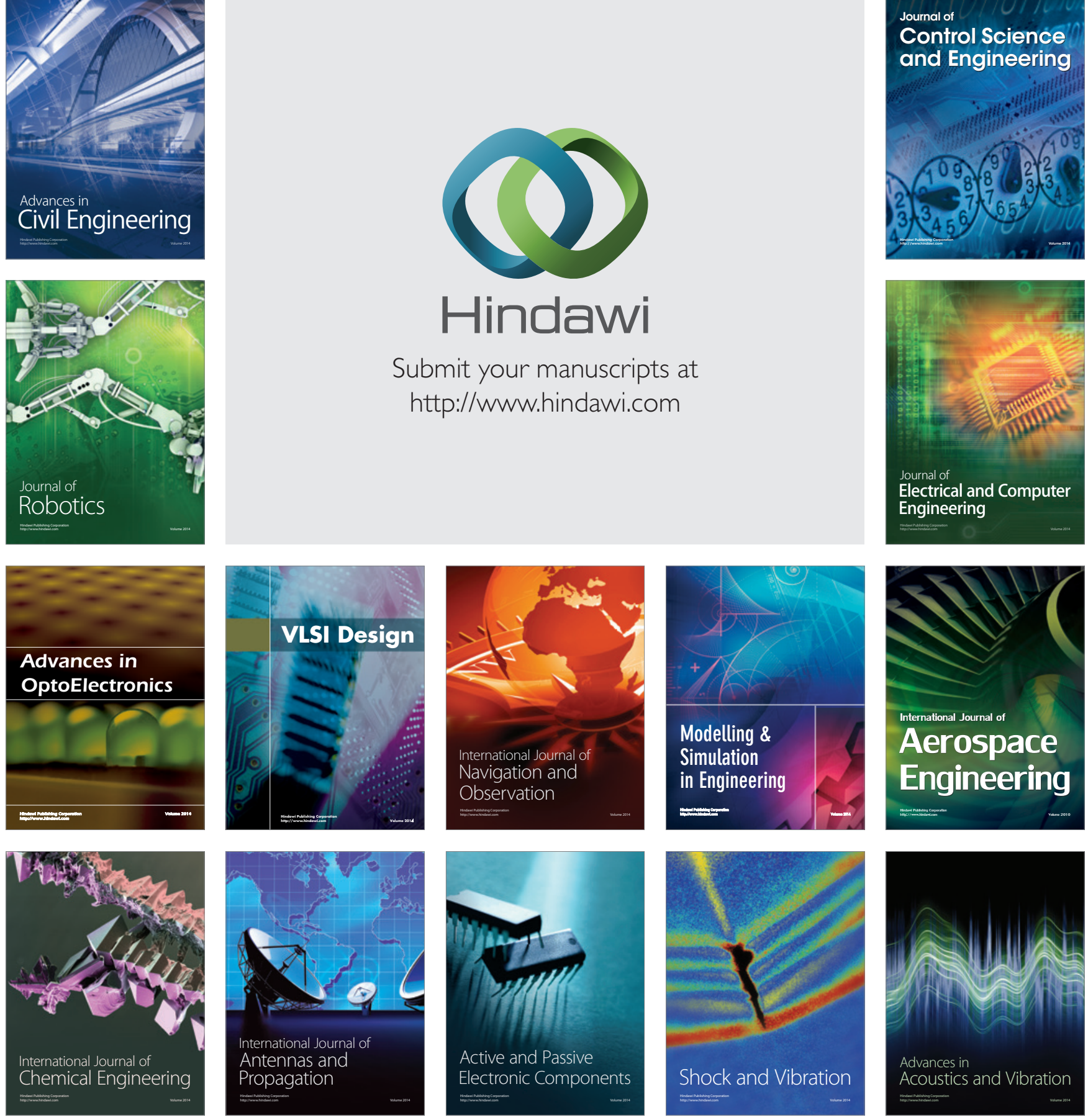